\title{
Relação entre os indicadores antropométricos do risco cardiometabólico e perfil socioeconômico de mulheres praticantes de atividade física
}

\author{
Relationship between anthropometric indicators of cardiometabolic risk and socioeconomic profile \\ of women practicing physical activity \\ Relación entre indicadores antropométricos de riesgo cardiometabólico y perfil socioeconómico de
} mujeres que practican actividad física

Recebido: 01/03/2021 | Revisado: 08/03/2021 | Aceito: 13/03/2021 | Publicado: 24/03/2021

Lazaro Batista Santos

ORCID: https://orcid.org/0000-0002-2239-136X

Faculdade Pitágoras Bacabal, Brasil

E-mail nutrilazarosantos@gmail.com

Maria Thairle dos Santos de Oliveira

ORCID: https://orcid.org/0000-0003-1630-9603

Faculdade Pitágoras Bacabal, Brasil

E-mail: thairlesantos@gmail.com

Luanda Sínthia Oliveira Silva Santana

ORCID: https://orcid.org/0000-0002-5053-4073

Faculdade Pitágoras Bacabal, Brasil

E-mail: lusossantana@gmail.com

Charlyan de Sousa Lima

ORCID: https://orcid.org/0000-0002-6100-0325 Faculdade Univates, Brasil

E-mail: charlyansl@yahoo.com.br

Silvana de Figueredo Alencar de Oliveira

ORCID: https://orcid.org/0000-0003-0979-5578

Faculdade Pitágoras Bacabal, Brasil

E-mail: 1asilvana@bol.com.br

Rayana Silva de Almeida

ORCID: https://orcid.org/0000-0003-3238-7361

Faculdade Pitágoras Bacabal, Brasil

E-mail: anayaralmeida@hotmail.com

Camila Guedes Borges de Araujo ORCID: https://orcid.org/0000-0001-5403-5258

Faculdade Pitágoras Bacabal, Brasil

E-mail: camilaguedes2@gmail.com

Bruna Cruz Magalhães

ORCID: https://orcid.org/0000-0002-1491-8694

Faculdade Pitágoras Bacabal, Brasil

E-mail: brunacruzmagalhaes@gmail.com

\begin{abstract}
Resumo
O estudo avaliou os indicadores antropométricos do risco cardiometabólico e sua relação com o perfil socioeconômico de mulheres praticantes de atividade física. Estudo transversal composto por 55 mulheres adultas de uma cidade do Nordeste brasileiro. O risco cardiometabólico foi determinado por meio das medidas de circunferência da cintura, índice de conicidade e índice de massa corporal. Além disso, foi realizada aplicação do Questionário de Frequência Alimentar com análise quantitativa através do software Dietsys versão 4.01. As participantes da pesquisa foram divididas segundo a classe econômica estabelecida pela Associação Brasileira de Empresas de Pesquisa e os dados foram analisados no software Graphpad prism 5.0®, com nível de significância em $p<0,05$ e com intervalo de confiança de 95\%. O trabalho foi aprovado pelo Comitê de Ética em Pesquisa do Hospital São Domingos e seguiu às normas do Conselho Nacional de Saúde da Resolução n ${ }^{\circ}$ 466/2012. As mulheres participantes do estudo tinham idade média entre 40 e 49 anos, e $80 \%$ apresentaram elevado índice de conicidade. O consumo calórico e de macronutrientes estava adequado, o risco cardiometabólico estava presente em todas as classes econômicas estudadas, porém as variáveis antropométricas eram semelhantes entre os grupos. Dessa forma, o estudo demonstrou que a prevalência de risco cardiometabólico foi elevada dentre as mulheres participantes do estudo e não houve associação entre os indicadores antropométricos e o perfil socioeconômico.
\end{abstract}

Palavras-chave: Antropometria; Doenças Cardiovasculares; Classe social; Mulheres. 


\begin{abstract}
The study evaluated the anthropometric indicators of cardiometabolic risk and their relationship with the socioeconomic profile of women practicing physical activity. Cross-sectional study with 55 adult women in the Northeast region of Brazil. Cardiometabolic risk was determined by waist circumference, conicity index and body mass index. In addition, the Food Frequency Questionnaire (QFA) was applied with quantitative analysis by the Dietsys software version 4.01. The research participants were divided according to the economic class established by the Brazilian Association of Research Companies (ABEP) and the data were analyzed using the Graphpad prism 5.0® software, with a significance level of $p<0.05$ and with a confidence interval of $95 \%$. The study was approved by the Research Ethics Committee of Hospital São Domingos and followed the rules of the National Health Council of Resolution No. 466/2012. The women participating in the study had an average age between 40 and 49 years, and $80 \%$ had a high conicity index. The caloric and macronutrient intake was adequate, the cardiometabolic risk was present in all the economic classes studied, but the anthropometric variables were similar between the groups. Thus, the study demonstrated that the prevalence of cardiometabolic risk was high among women participating in the study and there was no association between anthropometric indicators and socioeconomic profile.
\end{abstract}

Keywords: Anthropometry; Cardiovascular diseases; Social class; Women.

\title{
Resumen
}

El estudio evaluó indicadores antropométricos de riesgo cardiometabólico y su relación con el perfil socioeconómico de las mujeres que practican actividad física. Estudio transversal compuesto por 55 mujeres adultas de una ciudad del Nordeste de Brasil. El riesgo cardiometabólico se determinó midiendo la circunferencia de la cintura, el índice de ahusamiento y el índice de masa corporal. Además, se aplicó el Cuestionario de Frecuencia Alimentaria con análisis cuantitativo utilizando el software Dietsys versión 4.01. Como participantes de la investigación, fueron divididos según la clase económica otorgada por la Asociación Brasileña de Empresas de Investigación y los datos fueron transportados en el software Graphpad prism 5.0®, con un nivel de significancia de p <0.05 y con un intervalo de confianza del 95\%. El estudio fue aprobado por el Comité de Ética en Investigación del Hospital São Domingos y siguiendo las reglas del Consejo Nacional de Salud de la Resolución No. 466/2012. Las mujeres que participaron en el estudio tenían una edad promedio de entre 40 y 49 años y un índice de puesta a punto alto del $80 \%$. La ingesta calórica y de macronutrientes fue adecuada, el riesgo cardiometabólico estuvo presente en todas las clases atenuantes estudiadas, aunque las variables antropométricas fueron similares entre los grupos. Así, el estudio encontró que la prevalencia de riesgo cardiometabólico fue alta en las mujeres que participaron del estudio y no hubo asociación entre indicadores antropométricos y perfil socioeconómico.

Palabras clave: Antropometría; Enfermedades cardiovasculares; Clase social; Mujeres.

\section{Introdução}

Nas últimas décadas do século XX e início dos anos 2000, o Brasil passou por mudanças no cenário social, político e econômico, que incluiu melhoria dos níveis de educação, da cobertura dos serviços de saúde, aumento na renda familiar e alterações no padrão de empregos. Por sua vez, os serviços do setor primário da economia deram lugar aos trabalhos concentrados no setor secundário e, principalmente, no terciário (Batista \& Rissin, 2003).

Essas transições alteraram consideravelmente a vida e os hábitos da população, com importante impacto nos seus níveis de saúde. A partir disso, houve mudança na composição das dietas tradicionais ricas em fibras alimentares e grãos para uma alimentação com gorduras, rica em açúcares, grãos refinados, gordura animal e dietas proteicas, contribuindo para o aumento significativo dos fatores de riscos cardiometabólicos e das doenças crônicas (Lima et al., 2020).

Assim, foram observadas modificações das características sociodemográficas e econômicas da infância até a vida adulta, que indicam que fatores do início da vida influenciam o estado nutricional desta população, caracterizando obesidade em adultos. Isso se deve ao fato da trajetória desde a tenra idade e por toda a vida estarem relacionadas à atividade física, alimentação e drogas; e estes sofrem influência direta da condição socioeconômica e estão fortemente associados com o estado nutricional dos indivíduos (Bastos, 2016).

Sobre isso, houve mudanças no perfil de estatura, peso e composição corporal da população em todas as regiões brasileiras. A desnutrição diminuiu, enquanto que foi aumentada a prevalência de obesidade, ocasionando e constituindo fator de risco para aumento de demais doenças crônicas não transmissíveis, como hipertensão arterial, diabetes mellitus, alguns tipos de cânceres e doenças cardiovasculares (Conde \& Monteiro, 2014). 
A etiologia da obesidade é complexa e multifatorial, resultando da interação de genes, ambiente, estilo de vida e fatores emocionais, além da diminuição da pratica de atividade física e o aumento da ingestão calórica serem fatores ambientais determinantes para essa condição (ABESO, 2016). A obesidade caracteriza-se pelo acúmulo de tecido adiposo, regionalizado ou generalizado no tecido subcutâneo corpóreo e pode ser causada por doenças endócrino-metabólicas (obesidade endógena) ou alterações nutricionais (obesidade exógena), sendo, esta última, a mais prevalente (Longo et al., 2019).

A fim de avaliar a gordura central, as medidas antropométricas têm sido utilizadas devido as vantagens de aplicabilidade, baixo custo, inocuidade, simplicidade de utilização e interpretação, e podem ser aplicadas como indicadores de risco para doenças crônicas não transmissíveis (Lobatto et al., 2014).

$\mathrm{O}$ indicador antropométrico mais amplamente utilizado é o índice de massa corporal (IMC), que é calculado pela razão entre peso $(\mathrm{Kg})$ e altura $(\mathrm{m} 2)$. Por ser um indicador de obesidade generalizada, não sendo capaz de avaliar a gordura acumulada na região do abdome, deve ser aplicado juntamente com outros indicadores. Para isso, recomenda-se indicadores antropométricos de obesidade central, tais como perímetro da cintura, relação cintura/estatura e índice de conicidade, em substituição ou associados ao IMC, como parte da rotina do atendimento clínico e das estratégias de saúde pública (Machado et al., 2012).

Diante disso, Valdez propôs o Índice de Conicidade (IC), no início da década de 90 para avaliação da obesidade e distribuição da gordura corporal considerando que a obesidade central, mais do que a obesidade generalizada, está associada às doenças cardiovasculares, entre elas a hipertensão arterial sistêmica e a doença arterial coronariana (Pitanga \& Lessa, 2004).

Assim, a identificação de sobrepeso e obesidade, junto à avaliação antropométrica, pode contribuir sobremaneira para identificar precocemente a evolução e o risco das Doenças cardiovasculares (DCV). Diante disso, e com foco a medidas de prevenção, controle e melhoria da qualidade de vida, que este estudo teve como objetivo avaliar o risco cardiometabólico através das medidas antropométricas e sua relação com o perfil socioeconômico de mulheres praticantes de atividade física.

\section{Metodologia}

Trata-se de um estudo analítico descritivo e quantitativo, realizado em cidade do Nordeste Brasileiro. As participantes foram recrutadas no Serviço Social da Indústria (SESI) da cidade supracitada e as mesmas faziam parte do Projeto Saúde e Movimento. A amostra consistiu em 55 mulheres, sendo por conveniência e não probabilidade. Como critérios de inclusão adotou-se idade entre 25 e 59 anos, sexo feminino e assiduidade de pelo menos 2 dias ao projeto.

O protocolo de pesquisa foi submetido e aprovado pelo Comitê de Ética da Pesquisa do Hospital São Domingos (HSD) com Certificado de Apresentação e Apreciação Ética (CAAE) no 32829616.8.0000.5085. Todas as participantes em conformidade como o estudo assinaram um Termo de Consentimento Livre e Esclarecido (TCLE) após o esclarecimento sobre a natureza da investigação, em duas vias, um para o paciente e outra para o arquivo da pesquisa, obedecendo às normas do Conselho Nacional de Saúde contidas na Resolução nº 466/2012.

$\mathrm{O}$ método de entrevista com formulário estruturado foi aplicado pelos pesquisadores treinados para esse fim. Foram coletados dados antropométricos, como altura e peso para índice de massa corporal (IMC), e circunferência da cintura (CC) para cálculo do índice de conicidade (IC), segundo recomendações (Lohman, Roche \& Martorell, 1988) e classificação do estado nutricional pela Organização Mundial de Saúde (WHO, 2000).)

O consumo alimentar foi avaliado segundo Questionário de Frequência Alimentar (QFA) de caráter quantitativo e qualitativo (Ribeiro \& Cardoso, 2002). Esse questionário era composto de perguntas, as quais eram caracterizadas quanto ao tipo de alimento consumido, quantas vezes consumiu, e com que frequência foram ingeridos. Os dados obtidos foram exportados para planilhas do Microsoft Excel e analisados no Software DietSys versão 4.01. 
Aplicou-se o questionário da ABEP (2008) contendo perguntas fechadas acerca dos aspectos sociais, econômicos e demográficos. Obteve-se o somatório da pontuação obtida para cada paciente, e os mesmos foram classificados em classes econômicas (A1, A2, B1, B2, C1, C2, D, E (ABEP, 2008).

O índice de conicidade, por sua vez, foi determinado a partir dos dados antropométricos coletados para a aplicação na devida fórmula. Para o cálculo do Índice C foi utilizado à circunferência da cintura (m) dividida pela constante de 0,109 , vezes o resultado da raiz quadrada da divisão do peso $(\mathrm{kg})$ pela estatura $(\mathrm{m})$.

Figura 1 - Índice de Conicidade. (Pitanga \& Lessa, 2004).

$$
\text { Îndice } C=\frac{\text { Circunferência cintura }(\mathrm{m})}{0,109 \sqrt{\frac{\text { Peso corporal }(\mathrm{kg})}{\text { Estatura }(\mathrm{m})}}}
$$

A faixa teórica para indivíduos do gênero feminino do índice $\mathrm{C}$ é de 1,00 a 1,18, partindo de um cilindro perfeito para um cone duplo perfeito. $\mathrm{O}$ índice $\mathrm{C}$ aumenta de acordo com o acúmulo de gordura na região central do corpo, isto é, quanto mais próximo de 1,18, maior o acúmulo de gordura abdominal, aumentando o risco de doenças cardiovasculares (Valdez et al., 1993).

Os dados coletados foram integrados às planilhas do Excel 2010®. Em seguida à tabulação dos dados, os mesmos foram exportados para programa Graphpad prism 5.0® onde foi adotada a análise de variância (ANOVA) para comparação dos grupos, classificados de acordo com a classe econômica identificada pela ABEP, e adotou-se o nível de significância de 95\% $(\mathrm{p}<0,05)$. Os resultados foram apresentados em média e erro padrão em tabelas e gráficos.

\section{Resultados}

Dentre as 55 mulheres, a distribuição percentual demonstrou maioria na faixa etária de 40 a 49 anos de idade, correspondendo a um total de $36,4 \%$ das entrevistadas. A Tabela 1 apresenta a distribuição da faixa etária, raça, estado civil e classificação econômica das participantes do estudo, sendo caracterizada por mulheres pardas, casadas e pertencentes a classe $\mathrm{C} 2$. 
Tabela 1. Características socioeconômicas/demográficas da população estudada autodeclaradas.

\begin{tabular}{|c|c|c|}
\hline Variáveis & $\mathbf{N}$ & $\%$ \\
\hline \multicolumn{3}{|l|}{ Idade } \\
\hline $25-39$ & 19 & $34,5 \%$ \\
\hline 40- 49 & 20 & $36,4 \%$ \\
\hline $50-59$ & 16 & $29,1 \%$ \\
\hline \multicolumn{3}{|l|}{ Raça } \\
\hline Branca & 11 & $20 \%$ \\
\hline Preta & 10 & $18,2 \%$ \\
\hline Parda & 32 & $58,2 \%$ \\
\hline Amarela & 2 & $3,6 \%$ \\
\hline \multicolumn{3}{|l|}{ Estado civil } \\
\hline Solteira & 17 & $30,9 \%$ \\
\hline Casada & 30 & $54,6 \%$ \\
\hline Divorciada & 2 & $3,6 \%$ \\
\hline Viúva & 5 & $9,1 \%$ \\
\hline Outros & 1 & $1,8 \%$ \\
\hline \multicolumn{3}{|l|}{ Classificação Econômica } \\
\hline$\overline{\mathrm{B} 1}$ & 1 & $1,8 \%$ \\
\hline $\mathrm{B} 2$ & 10 & $18,1 \%$ \\
\hline $\mathrm{C} 1$ & 15 & $27,3 \%$ \\
\hline $\mathrm{C} 2$ & 20 & $36,4 \%$ \\
\hline D-E & 9 & $16,4 \%$ \\
\hline Total & 55 & $100 \%$ \\
\hline
\end{tabular}

Fonte: Dados da pesquisa.

A Tabela 2 demonstra a representação dos dados antropométricos dos indivíduos participantes desta pesquisa. As participantes da pesquisa têm CC caracterizando alto risco cardiovascular, IC superior a 1,18 e com classificação de elevado risco para o desenvolvimento de patologias metabólicas, e estado nutricional de sobrepeso considerando a média de IMC.

Tabela 2. Média e erro padrão dos indicadores antropométricos das mulheres do estudo.

\begin{tabular}{lc}
\hline \multicolumn{1}{c}{ VARIÁVEIS } & Média \pm Erro padrão \\
\hline Peso $(\mathrm{kg})$ & $68 \pm 12,69$ \\
Altura $(\mathrm{m})$ & $1,54 \pm 0,06$ \\
Circunferência da cintura $(\mathrm{cm})$ & $90,0 \pm 15,96$ \\
Índice de conicidade & $1,26 \pm 0,08$ \\
Índice de massa corporal $\left(\mathrm{kg} / \mathrm{m}^{2}\right)$ & $28,2 \pm 4,94$ \\
\hline
\end{tabular}

Fonte: Dados da pesquisa. 
A estimativa da frequência e ingestão de macronutrientes mediante avaliação de questionários de frequência alimentar (QFA) mostrou média de consumo energético de 2627,75 kcal, 53,59\% de consumo de carboidratos, 21,37\% de proteínas e 27,92\% de lipídios ingeridos (Tabela 3).

Tabela 3. Descrição do consumo médio de macronutrientes das mulheres do estudo.

\begin{tabular}{|c|c|c|c|c|}
\hline Variáveis & Quantidade (g) & $\begin{array}{c}\text { Quantidade } \\
\text { (kcal) }\end{array}$ & $\begin{array}{c}\text { \% da média de } \\
\text { nutrientes } \\
\text { referente ao } \\
\text { VEMC* }\end{array}$ & Recomendação \\
\hline \multicolumn{5}{|c|}{ Carboidratos } \\
\hline Média & 342,17 & 1368,7 & 52,09 & 50 a $60 \%$ \\
\hline \multicolumn{5}{|l|}{ Proteínas } \\
\hline Média & 136,45 & 545,8 & 20,77 & 15 a $20 \%$ \\
\hline \multicolumn{5}{|l|}{ Lípidios } \\
\hline Média & 79,25 & 713,25 & 27,14 & 25 a $30 \%$ \\
\hline
\end{tabular}

* Valor energético médio consumido (VEMC). Fonte: Dados da pesquisa.

A comparação entre as classes socioeconômicas e sua associação com as variáveis antropométricas está demonstrada nos Gráficos 1 a 3. Não houve diferença significante entre as classes sociais e os parâmetros de CC, IC e IMC. As mulheres da classe $\mathrm{C} 1$ apresentaram adequação de $\mathrm{CC}$ e sem RCV quando comparada as demais classes, e àquelas pertencentes a Classe DE apresentaram IC de 1,14 \pm 0,12 e sem risco cardiometabólico por essa variável.

Gráfico 1 - Confronto de valores de Média e erro padrão de circunferência da cintura (cm) e classe econômica das mulheres do estudo.

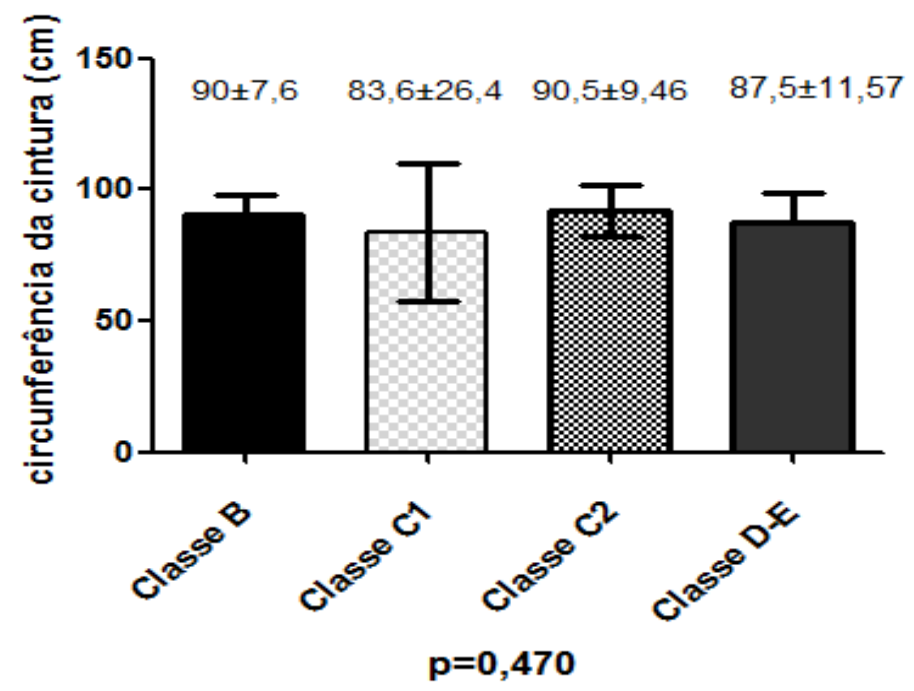

Fonte: Dados da pesquisa. 
Gráfico 2 - Detrimentos correlativos de média e erro padrão de Índice de Conicidade e perfil socioeconômico das mulheres do estudo.

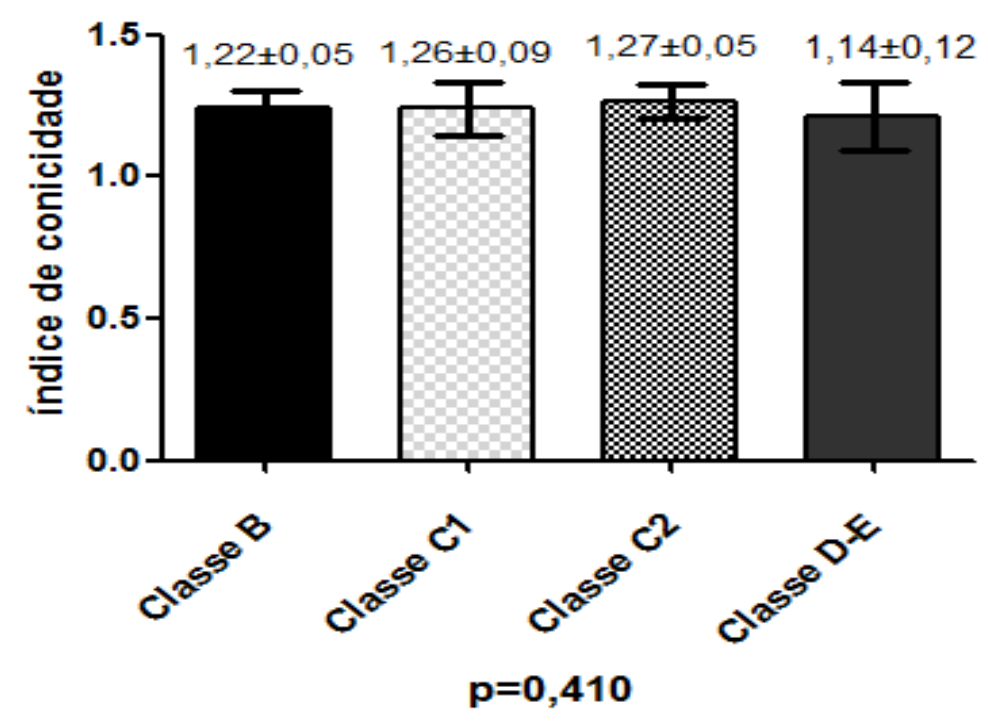

Fonte: Dados da pesquisa.

Gráfico 3 - Detrimentos correlativos dos valores de dispersão de Índice de Conicidade e perfil socioeconômico das mulheres do estudo.

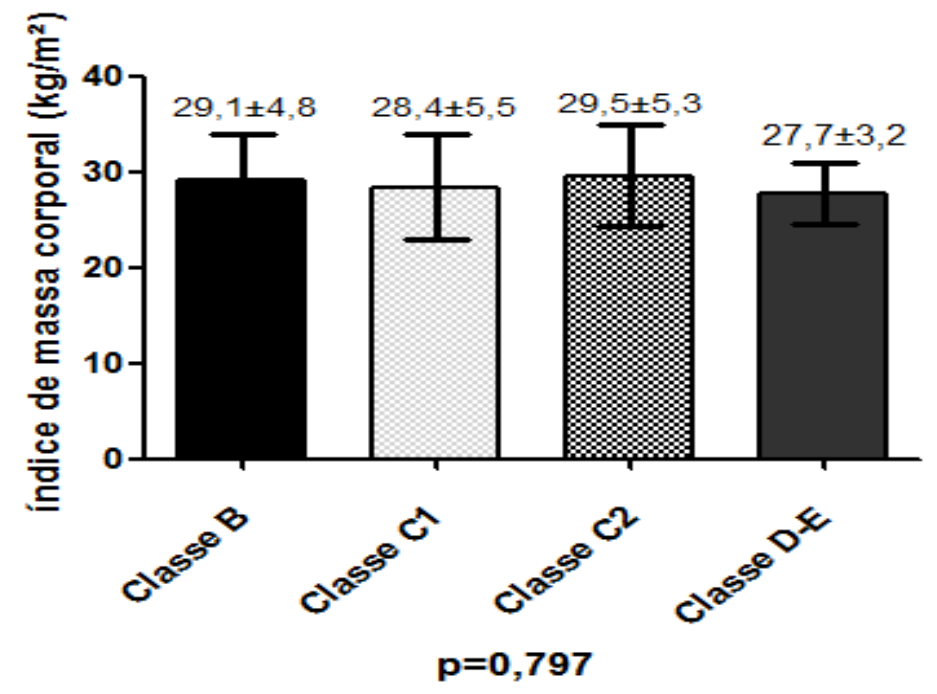

Fonte: Dados da pesquisa.

\section{Discussão}

Neste estudo foi observado que as participantes apresentaram risco cardiovascular segundo os dados de circunferência da cintura e índice de conicidade e estado nutricional de sobrepeso. Além disso, as medidas antropométricas cardiometabólicas não estavam associadas às diferentes classes econômicas, com adequada recomendação do consumo alimentar.

No que diz respeito ao percentual da faixa etária mais predominante representado na tabela 01 , os resultados deste estudo são consistentes com o pressuposto de que mulheres entre 40 e 49 anos podem ter risco cardiometabólico aumentado quando acompanhado de excesso de peso. Esse perfil pode ser atribuído ao climatério, que representa a transição do período 
reprodutivo para o não-reprodutivo, onde ocorrem manifestações de hipoestrogenismo, desencadeando, na maioria das mulheres, sintomas vasomotores, psicológicos, urogenitais, sexuais, distúrbios alimentares e distúrbios do sono. Esses, por sua vez, comprometem a qualidade de vida e favorecem o acumulo de peso comumente mais expressivo nessa fase (Gallon \& Wender, 2012).

Associado a idade e excesso de peso, as mulheres estudadas eram em sua maioria casadas, o que fortalece os resultados de risco cardiometabólico nessa população. Essas mulheres dispõem de afazeres domésticos e cuidados com os filhos e o cônjuge, e isso pode refletir em menor quantidade de tempo destinada aos cuidados com a própria saúde que, consequentemente, podem favorecer o acumulo de peso, entre outras consequências patológicas. Segundo Teichmann et al. (2006) em que em seu levantamento, demonstrou a ocorrência de sobrepeso e obesidade mais acentuada em mulheres casadas e de baixa escolaridade.

Os resultados de nível de escolaridade, renda familiar, e utensílios do lar, quando avaliados e classificados segundo classes econômicas, corroboram com o estudo de Linhares et al. (2012). Os autores explicam que classe econômicas mais baixas apresentam baixa/limitada acessibilidade aos meios básicos educacionais, permitindo que as mesmas se tornem impossibilitadas de distinguir a importância de uma alimentação adequada, assim como desconhecem os benefícios da prática de exercício físico. A ausência de informação e educação nutricional, segundo os autores, é ainda correlacionado com o risco para o desenvolvimento de comorbidades, como obesidade, hipertensão, dislipidemia, síndrome metabólica e câncer.

$\mathrm{O}$ aumento do risco e acometimento de patologias metabólicas podem ainda ser influenciadas pelo excesso de gordura provindo do sobrepeso e da obesidade. $\mathrm{O}$ acúmulo de peso corporal acelera a mobilização dos ácidos graxos pelo sistema porta devido ao aumento de lipólise, elevando a concentração dos ácidos graxos livres no fígado, e associando-se à maior incidência de distúrbios metabólicos, hormonais, inflamatórios e cardiovasculares, como intolerância à glicose, dislipidemia, hiperinsulinemia, resistência à insulina e estresse oxidativo, com redução crônica da disponibilidade do óxido nítrico (Ferreira, 2016).

Nessa abordagem, os resultados do presente estudo assemelham-se aos de Campos et al. (2016) e Da Silveira e Marques (2017), que obtiveram populações com índices elevados de acumulo energético, caracterizando excesso de peso e sua correlação com risco de desenvolvimento de distúrbios metabólicos.

Segundo Sawaya et. al. (2004)) uma possível explicação para esse excesso de peso e a relação com a estatura seja por uma ingestão alimentar insuficiente no início da vida, que ocasiona baixa estatura, pois levaria a um aumento na relação de cortisol: insulina, associado a baixos níveis de fator de crescimento insulina símile tipo 1 (IGF-). Como consequência, promove um reduzido ganho muscular e crescimento linear, além de prejudicar a oxidação de gordura e aumentar a razão cintura-quadril. Quando estas mudanças hormonais são combinadas a um aumento na ingestão alimentar e/ou diminuição/insuficiência da atividade física, o fenótipo baixa estatura associado à obesidade e suas co-morbidades pode ocorrer.

Além disso, através da CC pode-se mensurar a distribuição central da gordura na região abdominal, a fim de identificar potenciais riscos para determinadas co-morbidades. (21) De acordo com Silva et al. (2012) e Menezes et al. (2014), o acumulo de gordura na região abdominal está associado a uma menor concentração de adiponectina no organismo, e ocasiona prejuízo ao metabolismo da glicose e lipídios.

Concomitante a isso, o IC (Tabela 02), caracteriza as mulheres participantes do estudo em elevado risco para o desenvolvimento de patologias metabólicas. Uma vez que, segundo Pitanga \& Lessa (2004) o ponto de corte para mulheres compreende valores entre 1,0 e 1,18, ou seja, quanto maior o acúmulo de gordura na região central do corpo - mais próximo de 1,18 -, maior o risco para o desenvolvimento de doenças cardiovasculares.

O perfil de saúde de indivíduos está comumente ligado à sua posição socioeconômica (Bastos, 2016). Neste contexto, 
a atuação do nutricionista é fundamental na promoção e recuperação da saúde individual e coletiva onde, através da aplicação dos critérios diagnósticos para avaliação da obesidade, incluindo o índice de massa corpórea, análise dietética, circunferência da cintura e análise bioquímica reforçam a prevenção primária desta enfermidade e otimiza estratégias de intervenções positivas (Ulbrich et al., 2012).

A fim de avaliar a presença ou possível associação das variáveis da pesquisa e a condição socioeconômica, as participantes foram agrupadas segundo a classe econômica. Nossos resultados não demonstraram relação entre os parâmetros antropométricos analisados e a estratificação por classe social, sem diferença entre as classes econômicas (Gráficos 1, 2 e 3).

Apesar disso, é possível caracterizar os grupos de indivíduos com maior prevalência e risco para DCV. Nesse caso, as mulheres pertencentes as classes sociais B e C apresentaram inadequação aos valores de CC e IC, ao contrário da classe social D-E que demonstrou baixo IC e CC elevada. Vale ressaltar que se faz necessário que tais fatores que acarretam à obesidade e, consequentemente, o risco cardiovascular, sejam analisados continuamente de forma objetiva para que mais fatores sejam correlacionados a essas comobidades.

O excesso de peso se faz presente em diferentes grupos sociais, desde aqueles com baixo a alto valor econômico. Em comparação com a literatura nacional, entre 2000 e 2010, houve um progressivo aumento da prevalência de obesidade em todas as categorias de nível socioeconômico, principalmente nas classes mais baixas, em que a falta de acessibilidade dessas pessoas aos meios básicos, tonaram-se as mesmas vulneráveis (Rech et al., 2010).

Diversos estudos tem demonstrado a prevalência de sobrepeso e obesidade em diferentes grupos sociais com destaque ao maior número de indivíduos enquadrados em classes socioeconômicas $\mathrm{C}$, levando em conta que as demais classes se encontram vulneráveis para o acumulo de peso (Farias Junior et al., 2012; Teixeira \& Diaz, 2012; Malta et al., 2014). A prevalência do acumulo energético em indivíduos de classe social $\mathrm{C}$ tem sido associada e atribuída a fatores como número de filhos, falta de políticas de conscientização em suas cidades, baixa frequência de atividade física, consumo alimentar de alimentos de baixa qualidade e alta densidade energética, assim como fatores metabólicos que potencializam o risco para tais co-morbidades (Pinho et al., 2013).

Quanto à classe socioeconômica D-E, os dados obtidos demonstram risco para desenvolvimento de doenças cardiovasculares segundo a CC, fazendo referência a substancial vulnerabilidade de classes econômicas mais baixas para o desenvolvimento de co-morbidades, uma vez que segundo Ferreira \& Benicio (2015), as populações menos favorecidas tendem a não distinguir a importância dos cuidados a saúde, assim como não fazem distinção entre os benefícios obtidos através da pratica frequente de atividade física, onde a tendência de consumo alimentar concentra-se em alimentos de baixa qualidade e alta densidade energética, favorecendo assim o ganho de peso.

Observa-se então, que com as grandes mudanças atribuídas aos fatores epidemiológicos e estilo de vida da população, a prevalência de sobrepeso e obesidade cresceu exponecialmente, onde a mesma não faz distinção de cor, raça e muito menos de perfil socioeconômico, cabendo ao estado, aos profissionais da saúde, assim como aos próprios indivíduos uma tomada de atitude sobre tal situação (Carvalho et al., 2013).

\section{Considerações Finais}

As mulheres participantes do estudo, após estratificação em classes econômicas e sociais, apresentaram as medidas antropométricas que revelam estado nutricional de sobrepeso e risco cardiometabólico. Com isso, não há distinção entre as classes econômicas mais favorecidas e menos favorecidas neste quesito.

Este estudo apresentou limitações como: ausência de outros métodos de avaliação do consumo alimentar para identificar a variabilidade da dieta dessas mulheres, mensuração de parâmetros bioquímicos, além da limitada amostra de participantes desse estudo. Dessa forma, como estratégias investigativas futuras, sugere-se estudos que possam incluir esses 
pontos limitantes.

\section{Referências}

Associação Brasileira para o Estudo da Obesidade e da Síndrome Metabólica - ABESO. (2016). Diretrizes Brasileiras de Obesidade.

Associação Brasileira de Empresas de Pesquisa. Critério de Classificação Econômica Brasil. (2008). Critério de Classificação Econômica Brasil. IBOPE.

Batista Filho, M. \& Rissin A. (2003). Nutritional transition in Brazil: geographic and temporal trends. Cadernos de saúde pública, 19 , S181-91.

Bastos, J. L. (2016). Trajetória Socioeconômica e Obesidade em Adultos Residentes em Florianópolis, Santa Catarina [tese]. Santa Catarina: Universidade Federal de Santa Catarina; 2016.

Brito, A. N. M. de, et al (2020). Padrão alimentar e resistência à insulina em adolescentes. Research, Society and Development, 9 (12), 1-21.

Campos, J. R., Souza, M. F. C. de, Araújo, K. M. O., Olmo, S. G., Lima, A. A., \& Bergamini, C. P. D. O. (2016). O impacto do peso flutuante sobre fatores de risco cardiovascular em mulheres obesas. HU Revista, 41(3 e 4).

Carvalho, E. A. A., Simão, M. T. J., Fonseca, M. C., Andrade, R. G., Ferreira, M. S. G., Silva, A. F., Souza, I. P. R., \& Fernandes, B. S. (2013). Obesidade: aspectos epidemiológicos e prevenção. Revista Médica de Minas Gerais, 23(1): 74-82.

Conde, W, L. \& Monteiro, C. A. (2014). Nutrition transition and double burden of undernutrition and excess of weight in Brazil. The American journal of clinical nutrition, $100(6): 1617-22 \mathrm{~S}$.

Farias Júnior, J. C. de, et al (2012). Prática de atividade física e fatores associados em adolescentes no Nordeste do Brasil. Revista de Saúde Pública, 46(3), 505-515.

Ferreira, R. A. B., \& Benicio, M. H. D. (2015). Obesidade em Mulheres Brasileiras: associação com paridade e nível socioeconômico. Revista Pan Americana de Salud Publica, 37(4/5):337-42.

Ferreira, M. E. (2016). Síndrome metabólica e doenças cardiovasculares: do conceito ao tratamento. Arquivos Catarineneses de Medicina, 45(4): 95-109.

Gallon, C. W., \& Wender, M. C. O. (2012). Estado nutricional e qualidade de vida da mulher climatérica. Revista Brasileira de Ginecologia e Obstetrícia, 34(4), 175-183.

Linhares, R. da S., et al. (2012). Distribuição de obesidade geral e abdominal em adultos de uma cidade no Sul do Brasil. Cadernos de Saúde Pública, 28(3), 438-447.

Lobato, T. A. A., Torres, R. S., Guterres, A. S., Mendes, W. A. A., Maciel, A. P., Santos, F. C. C., Leal, S. V., Sato, A. L. S. A. (2014) Indicadores Antropométricos de Obesidade em Pacientes com Infarto Agudo do Miocárdio. Revista Brasileira de Cardiologia, $27(3)$ : $203-212$.

Longo, M., Zatterale, F., Naderi, J., Parrillo, L., Formisano, P., Raciti, G. A., Beguinot, F., \& Miele, C. (2019). Adipose Tissue Dysfunction as Determinant of Obesity-Associated Metabolic Complications. International journal of molecular sciences, 20(9), 2358.

Lohman, T. G., Roche, A. F., Martorell, R. (1988). Anthropometric Standardization Reference Manual. Human kinetics books.

Machado, S. P., Rodrigues, D. G. C., Viana, D. A. L., Sampaio, H. A. C. (2012) Correlação entre o Índice de Massa Corporal e Indicadores Antropométricos de Obesidade Abdominal em Portadores de Diabetes Mellitus Tipo 2. Revista Brasileira em Promoção da Saúde, 25 (4): 1-9.

Malta, D. C., et al. (2014). Prevalência de fatores de risco e proteção para doenças crônicas não transmissíveis em adultos: estudo transversal, Brasil 2012. Epidemiologia e Serviços de Saúde, 23(4), 609-622.

Menezes, T. N. de, et al. (2014). Obesidade abdominal: revisão crítica das técnicas de aferição e dos pontos de corte de indicadores antropométricos adotados no Brasil. Ciência \& Saúde Coletiva, 19(6), 1741-1754.

Obesity: preventing and managing the global epidemic. Report of a WHO consultation. (2000). World Health Organization technical report series, 894 , i-253.

Pinho, C. P. S., et al. (2013). Prevalência e fatores associados à obesidade abdominal em indivíduos na faixa etária de 25 a 59 anos do Estado de Pernambuco, Brasil. Cadernos de Saúde Pública, 29(2), 313-324.

Pitanga, F. J. G., \& Lessa, I. (2004). Sensibilidade e especificidade do índice de conicidade como discriminador do risco coronariano de adultos em Salvador, Brasil. Revista Brasileira de Epidemiologia, 7 (3): 259-269.

Rech, R. R., et al. (2010). Prevalência de obesidade em escolares de 7 a 12 anos de uma cidade Serrana do RS, Brasil. Revista Brasileira de Cineantropometria \& Desempenho Humano, 12(2), 90-97.

Ribeiro, A. B., \& Cardoso, M. A. (2002). Construção de um questionário de frequência alimentar como subsídio para programas de prevenção de doenças crônicas não transmissíveis. Revista de Nutrição, 15(2): 239-245.

Santos, S. F., \& Mattos, K. M. (2016). Circunferência da cintura e risco cardiovascular: um estudo em acadêmicos da área de saúde. Disciplinarum Scientia| Saúde, 12 (1): 93-102.

Sawaya, A. L., Martins, P. A., Grillo, L. P., \& Florêncio, T. T. (2004). Long-term effects of early malnutrition on body weight regulation. Nutrition reviews, $62, \mathrm{~S} 127-\mathrm{S} 133$. 
Research, Society and Development, v. 10, n. 3, e41310313422, 2021

(CC BY 4.0) | ISSN 2525-3409 | DOI: http://dx.doi.org/10.33448/rsd-v10i3.13422

Silva, L. R., Stefanello, J. M. F., Pizzi, J., Timossi, L. S., \& Leite, N. (2012). Aterosclerose subclínica e marcadores inflamatórios em crianças e adolescentes obesos e não obesos. Revista Brasileira de Epidemiologia, 15(4): 804-816.

Silveira, I. E., \& Marques, A. E. F. (2017). A importância da avaliação antropométrica para analisar os níveis de obesidade em crianças do ensino fundamental II na cidade de São João do Rio-PB. Revista de Pesquisa Interdisciplinar, 2 (2).

Teichmann, L., et al. (2006). Fatores de risco associados ao sobrepeso e a obesidade em mulheres de São Leopoldo, RS. Revista Brasileira de Epidemiologia, 9(3), 360-373.

Teixeira, A. D, \& Diaz, M. D. M. (2012). Obesidade e o sucesso no mercado de trabalho utilizando a POF 2008-2009. Revista Gestão \& Políticas Públicas, 1 (2).

Ulbrich, A. Z., et al. (2012). Probabilidade de hipertensão arterial a partir de indicadores antropométricos em adultos. Arquivos Brasileiros de Endocrinologia \& Metabologia, 56(6), 351-357.

Valdez, R., Seidell, J. C., Ahn, Y. I., \& Weiss, K. M. (1993). A new index of abdominal adiposity as an indicator of risk for cardiovascular disease. A crosspopulation study. International journal of obesity and related metabolic disorders: journal of the International Association for the Study of Obesity, 17(2), 7782. 